\title{
Preparation of free-standing films with sulfonyl group from 3-mercaptopropyl(trimethoxy)silane/1,2- bis(triethoxysilyl)ethane copolymer
}

\author{
Takahiro Gunji ${ }^{1}$, Yasunobu Shigematsu ${ }^{1}$, Takashi Kajiwara ${ }^{1}$ and Yoshimoto Abe ${ }^{1,2}$ \\ 3-Mercaptopropyl(trimethoxy)silane (MTMS)/1,2-bis(triethoxysilyl)ethane copolymer was synthesized by the hydrolytic \\ polycondensation of the two alkoxysilanes in the presence of hydrochloric acid as a catalyst under nitrogen flow. Free-standing \\ films were prepared by heating the copolymer at $80^{\circ} \mathrm{C}$ for 4 days. The films were dipped in a $30 \%$ hydrogen peroxide solution \\ at room temperature for 4 days. The sulfonated films were stable against hydrolysis and maintained their form at $150^{\circ} \mathrm{C}$. The \\ sulfonyl group content of the film at $150^{\circ} \mathrm{C}$ was $1.20 \mathrm{mmol} \mathrm{g}^{-1}$ when the composition of MTMS/1,2-bis(trimethoxysilyl)ethane \\ was 1:2.
}

Polymer Journal (2010) 42, 684-688; doi:10.1038/pj.2010.52; published online 23 June 2010

Keywords: free-standing film; hydrolytic polycondensation; MTMS; sol-gel process; 1,2-bis(triethoxysilyl)ethane

\section{INTRODUCTION}

Polysilsesquioxanes are defined as polysiloxanes, with the average formula $\left(\mathrm{RSiO}_{3 / 2}\right)_{n}$, and they are categorized as random, ladder or cage structures. ${ }^{1}$ Ladder and cage silsesquioxanes are known to be good target compounds in the fields of synthetic chemistry and materials chemistry because they have highly regulated structures. On the other hand, the isolation of random polysilsesquioxanes is difficult because these polymers are unstable against condensation and form gels easily.

The sol-gel process is often applied to the synthesis of polysilsesquioxanes. ${ }^{2}$ Linear polysiloxanes with alkoxy groups as a side chain are produced when alkoxysilanes are subjected to hydrolysis and condensation in the presence of an acid catalyst in a stepwise hydrolysis and condensation process. Silica gels with a three-dimensional structure are formed by the hydrolysis and condensation of alkoxysilanes in the presence of a basic catalyst by the random condensation of orthosilicic acid. In both cases, polysiloxanes with a wide molecular weight distribution are formed because the hydrolysis and polycondensation reactions occur in parallel.

The isolatable polysilsesquioxanes are synthesized by the hydrolytic polycondensation of alkoxysilanes under a nitrogen flow in the following systems: trimethoxy(methyl)silane, ${ }^{3,4}$ trimethoxy(vinyl) silane, ${ }^{3}$ 3-methacryloyloxypropyl(trimethoxy)silane $e^{5,6}$ and tetraethoxysilane. $^{7}$ Because these polysilsesquioxanes have high molecular weights and show high stability against gelation, bulk bodies, coating films and free-standing films can be prepared by a simple procedure.
For example, free-standing films with high flexibility were prepared by heating these polysilsesquioxanes gently. ${ }^{3}$ Because polysilsesquioxanes are composed of a siloxane unit $\mathrm{RSiO}_{3 / 2}$, polysilsesquioxanes are easily modified by introducing functional groups on the side chain. In addition, polysilsesquioxanes can be converted into organic/inorganic hybrid materials, which have various characteristics because of the functional groups on the side chain.

We have reported the preparation of 3-mercaptopropylpolysilsesquioxanes by the hydrolytic polycondensation of 3-mercaptopropyl (trimethoxy)silane (MTMS) and their transformation to free-standing films, ${ }^{8}$ which were expected to show ion conductivity by oxidation of mercapto groups. ${ }^{9-11}$ However, these oxidized free-standing films showed low water resistivity. One simple and easy technique to improve water resistance is the introduction of alkyl chains to the main chain of polysilsesquioxanes by the copolymerization of MTMS and 1,2-bis(triethoxysilyl)ethane (BTESE). In this paper, therefore, we report the preparation of MTMS/BTESE copolymers and their transformation to free-standing films according to Scheme 1 .

\section{EXPERIMENTAL PROCEDURE}

Reagents

MTMS (Shin-Etsu Chemical Industry, Tokyo, Japan), BTESE (Gelest, Morrisville, PA, USA), methanol (Kanto Chemical, Tokyo, Japan), tetrahydrofuran (THF) (Wako Pure Chemical, Osaka, Japan), hydrochloric acid (Wako Pure Chemical), 10\% dibutyltin dilaurate solution (Tokyo Kasei, Tokyo, Japan), $30 \%$

${ }^{1}$ Department of Pure and Applied Chemistry, Faculty of Science and Technology, Tokyo University of Science, Chiba, Japan and ${ }^{2}$ Department of Food Science, Faculty of Health and Nutrition, Tokyo Seiei College, Tokyo, Japan

Correspondence: Associate Professor T Gunji, Department of Pure and Applied Chemistry, Faculty of Science and Technology, Tokyo University of Science, 2641 Yamazaki, Noda, Chiba 278-8510, Japan.

E-mail: gunji@rs.noda.tus.ac.jp

Received 21 November 2009; revised 22 April 2010; accepted 4 May 2010; published online 23 June 2010 


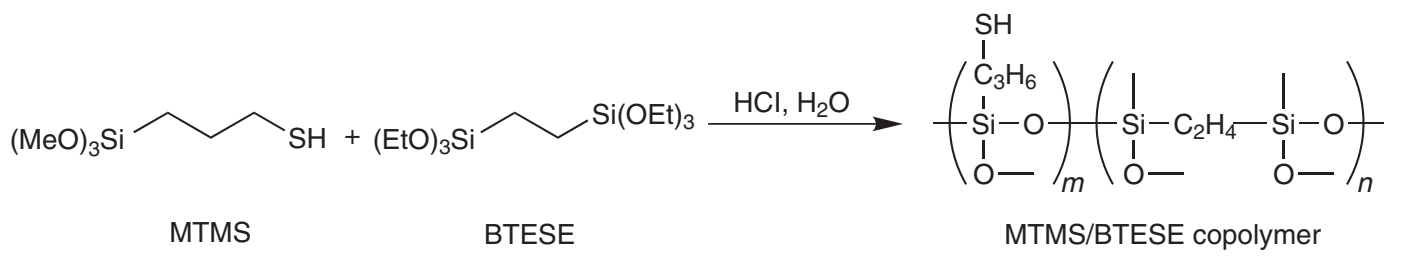

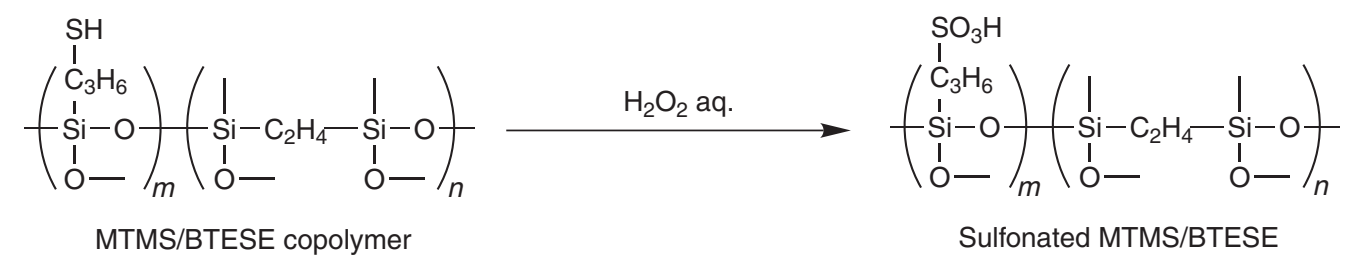

Scheme 1 Scheme for the preparation of sulfonated free-standing film.

hydrogen peroxide solution (Kanto Chemical), 10\% sodium chloride solution (Wako Pure Chemical) and sodium hydroxide (Kanto Chemical) were used as purchased.

\section{Preparation of MTMS/BTESE copolymer}

Into a $200-\mathrm{ml}$ four-necked flask equipped with nitrogen inlet and outlet tubes, $4.90 \mathrm{~g}(0.025 \mathrm{~mol})$ MTMS and $8.85 \mathrm{~g}(0.025 \mathrm{~mol})$ BTESE were charged and cooled in an ice bath for $10 \mathrm{~min}$. Nitrogen was introduced at a rate of $360 \mathrm{ml} \mathrm{min}^{-1}$, and $3.32 \mathrm{~g}(0.10 \mathrm{~mol})$ methanol, $0.125 \mathrm{~g}$ water and $0.967 \mathrm{~g}$ of $6 \mathrm{moll}^{-1}$ hydrochloric acid were then added in a dropwise manner, followed by stirring for $10 \mathrm{~min}$ at 150 r.p.m. After stirring for $10 \mathrm{~min}$ at room temperature, stirring was continued for $3 \mathrm{~h}$ at $70{ }^{\circ} \mathrm{C}$ to obtain MTMS/BTESE copolymer (No. 1) as a colorless highly viscous liquid.

Other MTMS/BTESE copolymers were prepared by changing the weights of MTMS and BTESE as follows: $3.33 \mathrm{~g}(0.017 \mathrm{~mol})$ MTMS and $12.04 \mathrm{~g}$ $(0.033 \mathrm{~mol})$ BTESE for MTMS/BTESE copolymer No. 2; $1.96 \mathrm{~g}(0.010 \mathrm{~mol})$ MTMS and $14.16 \mathrm{~g}(0.040 \mathrm{~mol})$ BTESE for MTMS/BTESE copolymer No. 3; and $0.98 \mathrm{~g}(0.005 \mathrm{~mol})$ MTMS and $15.93 \mathrm{~g}(0.045 \mathrm{~mol})$ BTESE for MTMS/ BTESE copolymer No. 4 . The amount of water was changed to maintain the molar ratio of water to silicon at 1:1.

\section{Preparation of MTMS/BTESE free-standing films}

A volume of $1 \mathrm{~g}$ of MTMS/BPESE copolymer was charged into a $20-\mathrm{ml}$ vial. A volume of $4 \mathrm{~g}$ of tetrahydrofuran was added to prepare a $20 \mathrm{wt} \%$ solution. After the addition of $0.4 \mathrm{~g}$ of $10 \%$ dibutyltin dilaurate solution, this solution was poured into a sharle made from polytetrafluoroethane and then allowed to rest at $80^{\circ} \mathrm{C}$ for 4 days.

\section{Sulfonation of MTMS/BTESE free-standing films}

MTMS/BTESE free-standing films were charged into a $200-\mathrm{ml}$ beaker. Thereafter, $30 \mathrm{ml}$ of $30 \%$ hydrogen peroxide solution was added and allowed to rest at room temperature for 3 days. At this point, these films were taken out of the solution and subjected to drying at room temperature.

\section{Heat treatment of MTMS/BTESE free-standing films}

MTMS/BTESE free-standing films were set in a glass sharle and heated with an electrical furnace for 1 day in air.

\section{Measurement and analysis}

${ }^{1} \mathrm{H},{ }^{13} \mathrm{C}$ and ${ }^{29} \mathrm{Si}$ Fourier-transformed nuclear magnetic resonance (NMR) spectra were obtained with a JEOL ECP-300 (JEOL, Tokyo, Japan). Chloroform- $d$ was used as a solvent.

Gel permeation chromatography was performed by a Shimadzu highperformance liquid chromatography system (Shimadzu, Kyoto, Japan) composed of an LD-10AD pump, two Polymer Laboratories (Church Stretton, UK) Mixed-D $250 \mathrm{~mm} \times 20 \mathrm{~mm}$ columns and a refractive index detector. Tetrahy- drofuran was used as the eluent $\left(1 \mathrm{ml} \mathrm{min}^{-1}\right)$. Molecular weight was calculated on the basis of standard polystyrenes.

The sulfonyl group content was determined by the following procedure: The sulfonated film was ground with an agate mortar. In a beaker, $50 \mathrm{mg}$ of powder was weighed and mixed with $50 \mathrm{ml}$ of $10 \mathrm{wt} \%$ sodium chloride solution. Stirring was continued gently overnight at room temperature. The solution was neutralized with $0.05 \mathrm{moll}^{-1}$ sodium hydroxide solution.

\section{RESULTS AND DISCUSSION}

\section{Results of the synthesis of MTMS/BTESE copolymers}

The synthesis of MTMS/BTESE copolymers was performed by the cohydrolytic polycondensation of MTMS and BTESE. Because MTMS and BTESE were hydrolyzed in the presence of an acid catalyst, silanols were stabilized to allow the condensation process to proceed. The sequence of MTMS/BTESE copolymers was simply controlled by the stability of the hydrolyzate and/or the silanols, which was affected by the steric hindrance around the silicon atoms. The steric hindrance around the silicon atom in MTMS was the same as that of BTESE because the ratio of methoxy and ethoxy groups in MTMS and BTESE was set to be equal by the transesterification reaction during the hydrolysis at $0{ }^{\circ} \mathrm{C}$, with condensation not being favored in this process. The MTMS/BTESE copolymers were, therefore, random copolymers consisting of MTMS and BTESE units. In addition, the hydrolytic polycondensation of alkoxysilanes was performed in the nitrogen flow to synthesize polysiloxanes with good reproducibility and to prevent gel formation during the formation and condensation of silanols. ${ }^{12-14}$

The results for MTMS/BTESE copolymers are summarized in Table 1. Before performing the syntheses shown in Table 1, a mixture of MTMS and BTESE was hydrolyzed with the molar ratio of water to silicon of 1.1:1 to yield a silica gel when the composition of MTMS/ BTESE was varied. In this work, therefore, the molar ratio of water to silicon was set to be 1:1 to synthesize polysilsesquioxane, with the molecular weight as large as possible. The molar ratio of MTMS/ BTESE was varied as 0.50:0.50 (No. 1), 0.34:0.66 (No. 2), 0.20:0.80 (No. 3) and 0.10:0.90 (No. 4). The yield was almost constant at $5.1-5.2 \mathrm{~g}$ because the amount of silicon atoms was set at $0.05 \mathrm{~mol}$, which resulted in the formation of the same amount of silanols. The weight-average molecular weight increased with increasing BTESE because BTESE functions as a hexafunctional monomer to form a siloxane network easily. In addition, the weight-average molecular weight and polydispersity increased drastically with increasing BTESE because more highly crosslinked copolymers were synthesized under conditions close to that of gel formation with increasing 
Table 1 Results for the synthesis of MTMS/BTESE copolymer ${ }^{a}$

\begin{tabular}{lcccccc}
\hline & \multicolumn{2}{c}{ Ratios of MTMS:BTESE } & & \multicolumn{2}{c}{ Molecular weight by $\mathrm{GPC}^{\mathrm{b}}$} \\
\cline { 2 - 3 } \cline { 6 - 6 } No & Mole & Weight per $g$ & & Yield per $g$ & $\mathrm{M}_{w} \times 10^{-3}$ & $\mathrm{M}_{w} \mathrm{M}_{n}$ \\
\hline 1 & $0.50: 0.50$ & $4.90: 8.85$ & 5.2 & 3.3 & 2.4 \\
2 & $0.34: 0.66$ & $3.33: 11.68$ & 5.1 & 4.4 & 2.9 \\
3 & $0.20: 0.80$ & $1.96: 14.16$ & 5.1 & 5.1 & 4.1 \\
4 & $0.10: 0.90$ & $0.98: 15.93$ & 5.1 & 14.1 & 8.6 \\
\hline
\end{tabular}

Abbreviations: BTESE, 1,2-bis(triethoxysilyl)ethane; GPC, gel permeation chromatography; MTMS, 3-mercaptopropyl(trimethoxy)silane.

Molar ratio: $\mathrm{HCl} / \mathrm{Si}=0.105$. Reagents: $\mathrm{MeOH} 3.32 \mathrm{~g}$. $\mathrm{H}_{2} \mathrm{O}: 0.125 \mathrm{~g} ; 6 \mathrm{~mol}^{-1} \mathrm{HCl}$ aqueous $0.967 \mathrm{~g}$. Temperature: $70^{\circ} \mathrm{C}$. Time: $3 \mathrm{~h} . \mathrm{N}_{2}$ flow rate: $360 \mathrm{ml} \mathrm{min}^{-1}$

ascale in operation: sum of the amount of substance for MTMS and BTESE: $0.05 \mathrm{~mol}$.

${ }^{\mathrm{b}}$ Calculated by GPC based on standard polystyrene.

amounts of hexafunctional monomer. MTMS/BTESE copolymers (Nos. 1-4) were soluble in acetone, tetrahydrofuran, chloroform and carbon tetrachloride, slightly soluble in hexane and insoluble in methanol and water.

The ${ }^{1} \mathrm{H}$ NMR spectra of MTMS/BTESE copolymers (Nos. 1-4) are shown in Figure 1. In the spectra of MTMS/BTESE copolymers, signals were observed at $0.72 \quad\left(\mathrm{Si}-\mathrm{CH}_{2}-\mathrm{CH}_{2}-\mathrm{Si}\right), \quad 0.78$ $\left(\mathrm{Si}-\mathrm{CH}_{2} \mathrm{CH}_{2} \mathrm{CH}_{2} \mathrm{SH}\right), \quad 1.25\left(-\mathrm{OCH}_{2} \mathrm{CH}_{3}\right), \quad 1.40 \quad\left(-\mathrm{CH}_{2} \mathrm{SH}\right), \quad 1.73$ $\left(-\mathrm{CH}_{2} \mathrm{CH}_{2} \mathrm{CH}_{2}-\right), 2.55\left(-\mathrm{CH}_{2} \mathrm{CH}_{2} \mathrm{SH}\right), 3.56\left(-\mathrm{OCH}_{3}\right)$ and 3.84 $\left(-\mathrm{OCH}_{2} \mathrm{CH}_{3}\right)$ p.p.m. The composition of MTMS and BTESE units in MTMS/BTESE copolymers was calculated on the basis of the proton integral ratio of the signals at 2.55 and 0.78 p.p.m., which showed good agreement with the compositions of MTMS and BTESE in the feed.

The ${ }^{29}$ Si NMR spectra of MTMS/BTESE copolymers (Nos. 1-4) are shown in Figure 2. The signals ascribed to $\mathrm{T}^{0}$ disappeared, whereas signals for $\mathrm{T}^{1}$ and $\mathrm{T}^{2}$ were observed at -48.2 to -53.9 and -55.1 to -62.2 p.p.m., respectively. The signals due to $\mathrm{T}^{3}$ were not observed $\left(\mathrm{T}^{n}\right.$ denotes the structure $\mathrm{Si}(\mathrm{OSi})_{n}(\mathrm{OMe})_{3-n}(n=0-3)$.) The ratio of siloxane units is summarized in Table 2. The ratios of $\mathrm{T}^{1}: \mathrm{T}^{2}$ were calculated as 39:61 (No. 2), 43:57 (No. 3), 44:56 (No. 3) and 42:58 (No. 4). The percentages of siloxane bonding were calculated as 54 (No. 1), 52 (No. 2), 52 (No. 3) and 53\% (No. 4), according to the equation $\sum_{n}^{0-3}\left(n \times \mathrm{T}^{n} / 3\right)(\%)$, where $\mathrm{T}^{n}$ denotes the percentages of $\mathrm{T}^{n}$ units in the ${ }^{29} \mathrm{Si} \mathrm{NMR}$ spectrum. These data indicate that MTMS/ BTESE copolymers are mainly composed of $\mathrm{T}^{2}$ units, and the composition was almost constant. Because the molar ratio of water to silicon atoms was maintained at 1:1, the degree of hydrolysis for each silicon atom was almost constant. The molecular weight of the MTMS/BTESE copolymer did increase, however, because BTESE is a hexafunctional alkoxysilane that forms a siloxane network more easily than MTMS.

\section{Results of the preparation of self-standing films from MTMS/ BTESE copolymers and their sulfonation}

Self-standing films were successfully prepared by aging MTMS/BTESE copolymers (Nos. 1-4) in a sharle made from polytetrafluoroethane in the presence of dibutyltin dilaurate. When the solution of MTMS/ BTESE copolymers was heated at $80^{\circ} \mathrm{C}$, solvents were evaporated after 1 day to yield a viscous liquid. After heating for 4 days, MTMS/BTESE copolymers solidified to form transparent and flexible free-standing films that peeled from the polytetrafluoroethane sharle. Because dibutyltin dilaurate is known as a catalyst to accelerate the formation of siloxane networks, ${ }^{15,16}$ the formation of free-standing films was carried out by the further hydrolytic condensation of methoxy groups

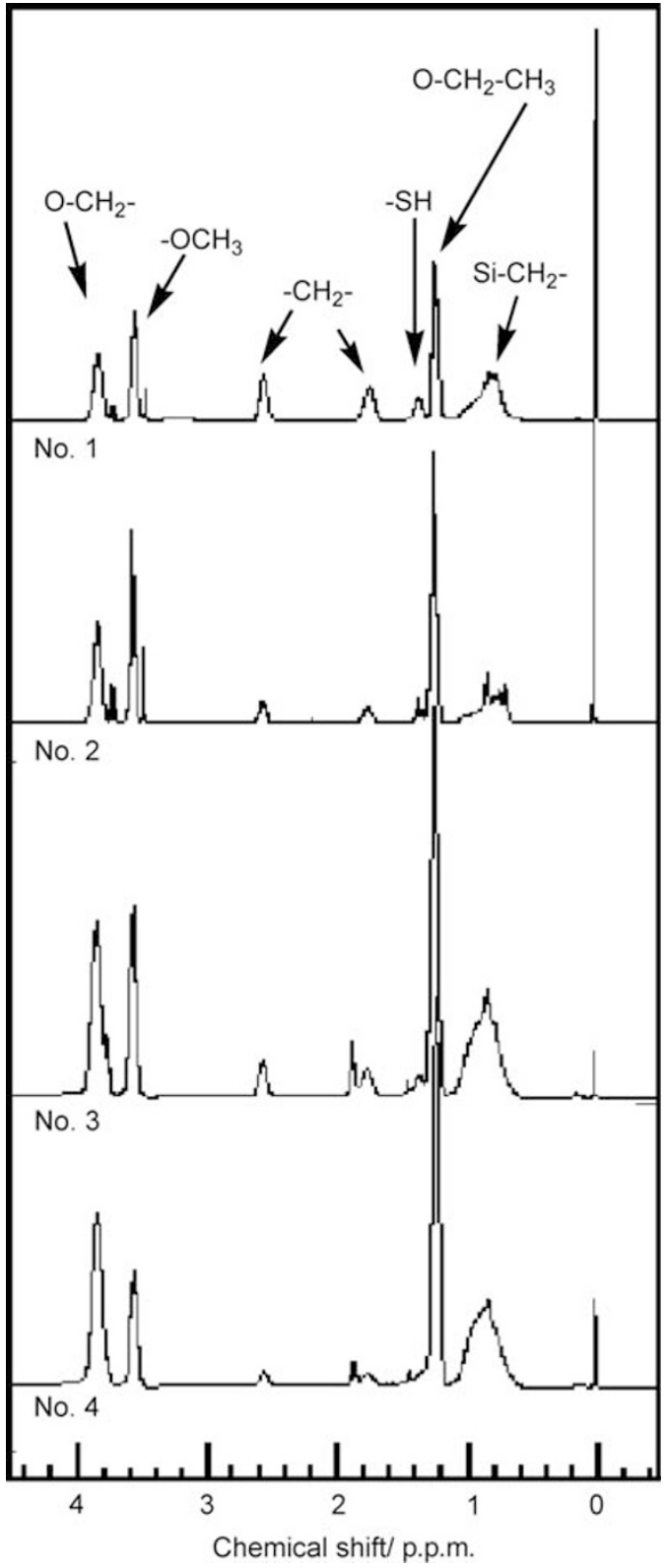

Figure $1{ }^{1} \mathrm{H}$ NMR spectra of MTMS/BTESE copolymers.

in MTMS/BTESE copolymers. Indeed, no gelation was observed when MTMS/BTESE copolymers were heated in the absence of dibutyltin dilaurate.

Self-standing films were immersed in a $30 \%$ hydrogen peroxide solution to produce sulfonated films. Before the oxidation of selfstanding films, we tried the oxidation of mercapto groups by hydrogen peroxide to show the oxidation of mercapto groups to sulfonyl groups and no formation of other intermediates such as sulfenyl and sulfinyl groups. Therefore, we used hydrogen peroxide in this process. The results of the preparation of sulfonated free-standing films are summarized in Table 3 . The sulfonyl group contents of free-standing films prepared from MTMS/BTMSE copolymer No. 2 were 0.85 (1 day), 1.10 (2 days), 1.23 (3 days) and $1.23 \mathrm{mmolg}^{-1}$ (4 days), which showed that sulfonation proceeded by 3 days. As a result, the reaction time and/or immersing time was set to be 3 days. A photograph of sulfonated free-standing film prepared from 


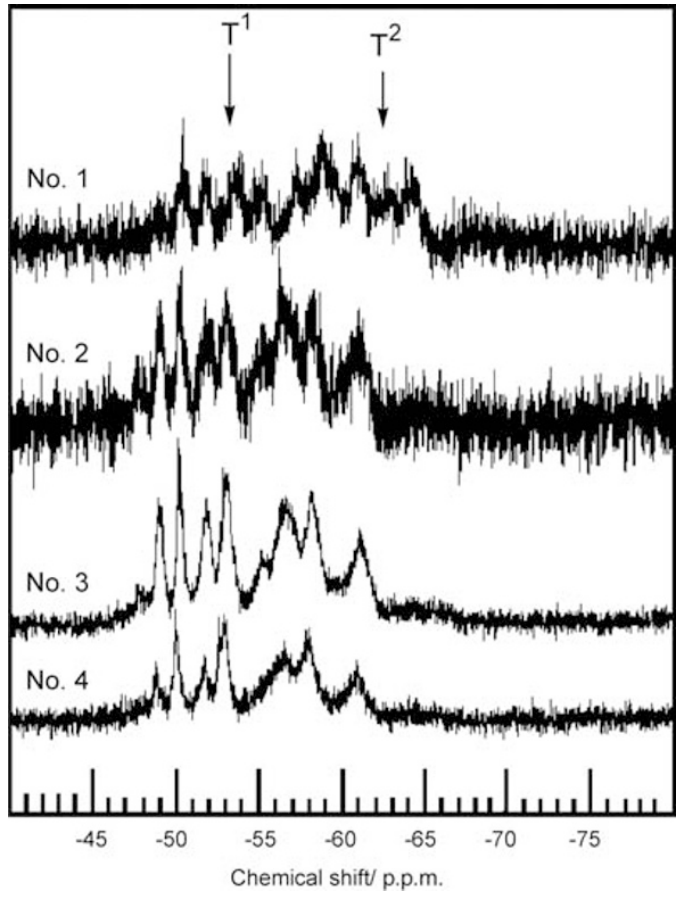

Figure $2{ }^{29} \mathrm{Si}$ NMR spectra of MTMS/BTESE copolymers.

Table 2 Ratio of siloxane units of MTMS/BTESE copolymer

\begin{tabular}{llllll}
\hline & \multicolumn{5}{c}{ Peak ratio of T unit } \\
\cline { 2 - 5 } No. & $T^{0}$ & $T^{1}$ & $T^{2}$ & $T^{3}$ & $D C^{\text {a }}$ \\
\hline 1 & 0 & 39 & 61 & 0 & 54 \\
2 & 0 & 43 & 57 & 0 & 52 \\
3 & 0 & 44 & 56 & 0 & 52 \\
4 & 0 & 42 & 58 & 0 & 53 \\
\hline
\end{tabular}

Abbreviations: BTESE, 1,2-bis(triethoxysilyl)ethane; MTMS, 3-mercaptopropyl(trimethoxy)silane. DC $(\%)=\sum\left(T^{n}(\%) \times n / 3\right)$.

a Degree of crosslinking of siloxane bonding.

Table 3 Results for the sulfonation ${ }^{\mathrm{a}}$ of MTMS/BTESE films

\begin{tabular}{llcc}
\hline & & \multicolumn{2}{c}{ Sulfonyl group content per mmol per $g$} \\
\cline { 3 - 4 } No. & State & Observed & Calculated \\
\hline 1 & Broken into pieces & $1.35(38.4 \%)$ & 3.51 \\
2 & Film was a few cracking & $1.23(47.9 \%)$ & 2.57 \\
3 & Free-standing film & $0.83(50.9 \%)$ & 1.63 \\
4 & Free-standing film & $0.44(50.6 \%)$ & 0.87 \\
\hline
\end{tabular}

Abbreviations: BTESE, 1,2-bis(triethoxysilyl)ethane; MTMS, 3-mercaptopropyl(trimethoxy)silane. aTemperature: room temperature Time: 3 days. Reagent: $30 \mathrm{wt} \% \mathrm{H}_{2} \mathrm{O}_{2}$ aqueous.

MTMS/BTESE copolymer No. 2 is shown in Figure 3. A transparent and flexible film with some cracks was prepared. A laboratory-made apparatus found the electron conductivity of this film in water at $25^{\circ} \mathrm{C}$ to be $0.0067 \mathrm{~S} \mathrm{~cm}^{-1}$.

The transparent and flexible film broke into pieces with MTMS/ BTESE copolymer No. 1, whereas continuous films were prepared from MTMS/BTESE copolymers Nos. 2-4, as shown in Figure 3.

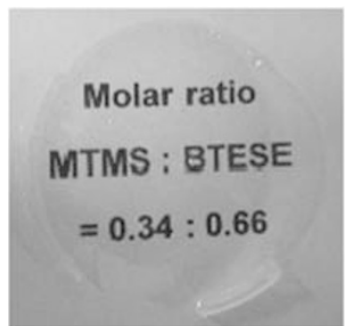

Figure 3 Photograph of sulfonated MTMS/BTESE copolymer (No. 2) film.

Table 4 Temperature change of sulfonated MTMS/BTESE film ${ }^{a}$

\begin{tabular}{llc}
\hline Temperature $\left({ }^{\circ} \mathrm{C}\right)$ & Color & $\begin{array}{c}\text { Sulfonyl group content } \\
\text { per mmol per } g\end{array}$ \\
\hline Room temperature & Colorless & 1.23 \\
80 & Colorless & 1.20 \\
100 & Pale yellow & 1.20 \\
120 & Yellow & 1.20 \\
150 & Yellow & 1.20 \\
\hline
\end{tabular}

Abbreviations: BTESE, 1,2-bis(triethoxysilyl)ethane; MTMS, 3-mercaptopropyl(trimethoxy)silane. a Sample: No.2 (sulfonyl group content: $1.23 \mathrm{mmol} \mathrm{g}^{-1}$ ).

These films were stable against humidity and retained their forms because of the low water-uptake ability and/or hydrophilicity. The sulfonyl group contents were 1.35 (No. 1), 1.23 (No. 2), 0.83 (No. 3) and $0.44 \mathrm{mmolg}^{-1}$ (No. 4), which decreased with the decreasing content of MTMS in the copolymer. On the other hand, the percentages of sulfonation, which were calculated as the ratio of observed and calculated sulfonyl group contents, were 38.4 (No. 1), 47.9 (No. 2), 50.9 (No. 3) and 50.6\% (No. 4), which suggests that half of the mercapto groups were sulfonated. In the sulfonation of mercapto groups, the mercapto groups on the surface of the film would be sulfonated first because the self-standing film is hydrophobic. The cracks would be formed by the increasing volume due to the sulfonation of mercapto groups and the degradation of the siloxane network catalyzed by sulfonic acid. The content of sulfonyl groups was varied by the composition of MTMS in the copolymer.

The sulfonyl group contents after heating for 1 day are summarized in Table 4. Sulfonated films were heated at $80,100,120$ and $150{ }^{\circ} \mathrm{C}$ for 1 day, and the sulfonyl group content was determined. The color of the film changed from colorless to pale yellow and then to yellow by increasing the heating temperature, which was due to the oxidation of organic groups in the films. The sulfonyl group contents were 1.23 $\left(80{ }^{\circ} \mathrm{C}\right), 1.20\left(100{ }^{\circ} \mathrm{C}\right), 1.20\left(120^{\circ} \mathrm{C}\right)$ and $1.20 \mathrm{mmolg}^{-1}\left(150^{\circ} \mathrm{C}\right)$, which were almost constant in comparison with the value at room temperature $\left(1.23 \mathrm{mmolg}^{-1}\right)$. These data show that these sulfonated self-standing films would work at $150{ }^{\circ} \mathrm{C}$ as a proton conductive film.

\section{CONCLUSION}

MTMS/BTESE copolymer was synthesized by the hydrolytic polycondensation of the two alkoxysilanes in the presence of hydrochloric acid as a catalyst under nitrogen flow. The molar ratio of MTMS:BTESE varied, and was 0.50:0.50, 0.34:0.66, 0.20:0.80 and 0.10:0.90. Free-standing films were prepared by heating copolymers at $80{ }^{\circ} \mathrm{C}$ for 4 days. The films were dipped in a $30 \%$ hydrogen peroxide solution at room temperature for 4 days. The sulfonated films were stable against hydrolysis and maintained their form at $150{ }^{\circ} \mathrm{C}$. The sulfonyl group content of the films at $150^{\circ} \mathrm{C}$ was $1.20 \mathrm{mmol} \mathrm{g}^{-1}$ when the composition of MTMS:BTESE was 0.34:0.66. 
1 Abe, Y. \& Gunji, T. Oligo and polysiloxanes. Prog. Polym. Sci. 29, 149-182 (2004).

2 Wright, J. D. \& Sommerdijk, N.A.J.M. Sol-Gel Materials. Chemistry and Applications (Gordon and Breach Science Publishers, Amsterdam, 2001).

3 Takamura, N., Gunji, T., Hatano, H. \& Abe, Y. Preparation and properties of polysilsesquioxanes: polysilsesquioxanes and flexible thin films by acid-catalyzed controlled hydrolytic polycondensation of methyl- and vinyltrimethoxysilane. J. Polym. Sci. Part A: Polym. Chem. 37, 1017-1026 (1999).

4 Gunji, T., Okonogi, M., Sakan, T., Takamura, N., Arimitsu, K. \& Abe, Y. Preparation and properties of organic-inorganic hybrid gel films based on polyvinylsilsesquioxane synthesized from trimethoxy(vinyl)silane. Appl. Organometal. Chem. 17, 580-588 (2003).

5 Abe, Y., Honda, Y. \& Gunji, T. Preparation and properties of silicon-containing polymer hybrids from 3-methacryloxypropyltrimethoxysilane. Appl. Organometal. Chem. 12, 749-753 (1998).

6 Gunji, T., Makabe, Y., Takamura, N. \& Abe, Y. Preparation and characterization of organic-inorganic hybrids and coating films from 3-methacryloxypropylpolysilsesquioxane. Appl. Organometal. Chem. 15, 683-692 (2001).

7 Abe, Y., Shimano, R., Arimitsu, K. \& Gunji, T. Preparation and properties of high molecular weight polyethoxysiloxanes stable to self-condensation by acid-catalyzed hydrolytic polycondensation of tetraethoxysilane. J. Polym. Sci. Part A: Polym. Chem. 41, 2250-2255 (2003).
8 Gunji, T., Shigematsu, Y., Abe, Y., Fujita, S. \& Inagaki, S. Preparation of free-standing films from 3-mercaptopropylpolysilsesquioxane. Kobunshi Ronbunshu 64, 705-707 (2007).

9 Marschall, R., Rathouskỳ, J. \& Wark, M. Ordered functionalized silica materials with high proton conductivity. Chem. Mater. 19, 6401-6407 (2007).

10 Marschall, R., Bannat, I., Caro, J. \& Wark, M. Proton conductivity of sulfonic acid functionalised mesoporous materials. Microporous Mesoporous Mater. 99, 190-196 (2007).

11 McKeen, J. C., Yan, Y. S. \& Davis, M. E. Proton conductivity of acid-functionalized zeolite Beta, MCM-41, and MCM-48: effect of acid strength. Chem. Mater. 20, 5122-5124 (2008).

12 Sakka, S. \& Kamiya, K. Glasses from metal alcoholates. J. Non-Cryst. Solids. 42, 403-421 (1980).

13 Brinker, C. J. \& Scherer, G. W. Sol-Gel Science. The Physics and Chemistry of Sol-Gel Processing (Academic Press, San Diego, 1989).

14 Brinker, C. J., Keefer, K. D., Schaefer, D. W., Assink, R. A., Kay, B. D. \& Ashley, C. S. Sol-gel transition in simple silicates II. J. Non-Crystal. Solids 63, 45-59 (1984).

15 Toynbee, J. Silane crosslinking of polyolefins: observations on the tin catalyst employed. Polymer 35, 438-440 (1994).

16 Adachi, K., Hirano, T., Fukuda, K. \& Nakamae, K. Controllable silane water-crosslinking kinetics and curability of ethylene-propylene copolymer by amine compounds. Macromol. React. Eng. 1, 313-320 (2007). 